\title{
Classification and clinical features of headache patients: an outpatient clinic study from China
}

\author{
Yunfeng Wang $\cdot$ Jiying Zhou $\cdot$ Xiaoping Fan $\cdot$ \\ Xuelian Li · Li Ran · Ge Tan · Lixue Chen • \\ Kuiyun Wang • Bowen Liu
}

Received: 14 March 2011 / Accepted: 9 June 2011/Published online: 9 July 2011

(C) The Author(s) 2011. This article is published with open access at Springerlink.com

\begin{abstract}
This study aimed to analyze and classify the clinical features of headache in neurological outpatients. A cross-sectional study was conducted consecutively from March to May 2010 for headache among general neurological outpatients attending the First Affiliated Hospital of Chongqing Medical University. Personal interviews were carried out and a questionnaire was used to collect medical records. Diagnosis of headache was according to the International classification of headache disorders, 2nd edition (ICHD-II). Headache patients accounted for $19.5 \%$ of the general neurology clinic outpatients. A total of 843 $(50.1 \%)$ patients were defined as having primary headache, $454(27 \%)$ secondary headache, and $386(23 \%)$ headache not otherwise specified (headache NOS). For primary headache, 401 (23.8\%) had migraine, 399 (23.7\%) tensiontype headache (TTH), $8(0.5 \%)$ cluster headache and 35 (2.1\%) other headache types. Overall, migraine patients suffered (1) more severe headache intensity, (2) longer than 6 years of headache history and (3) more common analgesic medications use than TTH ones $(p<0.001)$.TTH patients had more frequent episodes of headaches than migraine patients, and typically headache frequency exceeded 15 days/month $(p<0.001) ; 22.8 \%$ of primary headache patients were defined as chronic daily headache. Almost $20 \%$ of outpatient visits to the general neurology department were of headache patients, predominantly primary headache of migraine and TTH. In outpatient headaches, more attention should be given to headache intensity
\end{abstract}

Y. Wang $\cdot$ J. Zhou $(\bowtie) \cdot$ X. Fan $\cdot$ X. Li $\cdot$ L. Ran · G. Tan L. Chen $\cdot$ K. Wang $\cdot$ B. Liu

Department of Neurology, The First Affiliated Hospital of Chongqing Medical University, Chongqing 400016, China e-mail: zhoujiying@hospital-cqmu.com and duration of headache history for migraine patients, while more attention to headache frequency should be given for the TTH ones.

Keywords Outpatient · Headache ·

Cross-sectional study $\cdot$ Clinical feature $\cdot$ Migraine

$\begin{array}{ll}\text { Abbreviations } & \\ \text { CDH } & \text { Chronic daily headache } \\ \text { CM } & \text { Chronic migraine } \\ \text { CTTH } & \text { Chronic tension-type headache } \\ \text { MOH } & \text { Medication-overuse headache } \\ \text { TTH } & \text { Tension-type headache } \\ \text { NDPH } & \text { New daily persistent headache } \\ \text { ED } & \text { Emergency department } \\ \text { Headache NOS } & \begin{array}{l}\text { Headache not otherwise specified } \\ \text { ICHD-II }\end{array} \\ & \begin{array}{l}\text { International classification of headache } \\ \text { disorders, 2nd edition }\end{array}\end{array}$

\section{Introduction}

Headache is experienced by the majority of the population and has a major impact on public health. Worldwide, the condition has been ranked among the ten most disabling conditions by the World Health organization (WHO). In the global population, the prevalence of active headache disorder is $47 \%$, migraine $10 \%$, tension-type headache (TTH) $38 \%$ and chronic headache 3\%; the disability attributable to TTH is larger worldwide than that due to migraine [1]. Furthermore, headache is the most common neurological symptom presented by patients to general practitioners and neurologists [2].

Although headache disorders affect the majority of the adult population, most headaches can be self-managed and 
do not require medical intervention. Patients who consult doctors have different clinical features and the distribution of headache types seen in the clinic differs from population-based studies. A study by an ED in Greece [3] reported that TTH was the most frequent diagnosis, followed by secondary headache and migraine. In a clinical study from a headache center in east Hungary [4], migraine was the most common headache, while TTH patients reported more severe disability than migraines. A study from Asia [5] also showed that migraine was the most common headache diagnosis in neurological services. A population survey in Chile [6] indicated that migraineurs were more likely to consult doctors than TTH patients. A study from Denmark [7] reported that $56 \%$ of migraine patients and $16 \%$ of patients with TTH were seen by their family practitioner, whereas $16 \%$ of migraine and $4 \%$ of TTH patients sought specialist consultation.

Nevertheless, little is known about the distribution of different types of headache, including primary and secondary headaches, in outpatient departments where most patients in China first present. The aim of this survey was therefore to record the demographic data of patients attending for consultation of headache treatment in the general neurological outpatient department of a tertiary care hospital. Because comparison of the clinical characteristics of the different headache types could aid the development of headache treatment guidelines for public health intervention, the study also analyzed the clinical features of patients with migraine and TTH.

\section{Patients and methods}

A cross-sectional study was conducted consecutively from March to May 2010 for headache among patients attending the neurological outpatient department at the First Affiliated Hospital of Chongqing Medical University. Each participant was interviewed by a qualified headache doctor and completed a self-administered questionnaire. Diagnosis was performed by doctors participating in the patient interviews and the diagnosis of $\mathrm{CDH}$ was confirmed by a headache specialist (corresponding author). The questionnaire was in three sections. The first included demographic details and past health status. The second comprised a headache profile, including duration of headache history $(<1,1-3,4-6,>6$ years), attack frequency (average number of headache days per month: $<1,2-4,5-14$, or $\geq 15$ days/month) and severity using a $0-10$ visual analog scale (VAS); pulsating, pressing/tightening or other headaches; presence or absence of headache aggravation from climbing stairs or routine physical activities; location (unilateral, bilateral or orbital), duration of headache attacks ( $<30 \mathrm{~min}, 30 \mathrm{~min}$ to $2 \mathrm{~h}, 2-4 \mathrm{~h}, 4-72 \mathrm{~h}$ or $>3$ days) and accompanying symptoms (presence or absence of nausea, vomiting, photophobia or phonophobia, and clinical signs of cranial sympathetic dysfunction such as conjunctival injection, lacrimation, nasal congestion, rhinorrhoea, forehead and facial sweating, miosis, ptosis, eyelid edema). In addition, four major migraine aura symptoms (visual, sensory, motor and speech symptoms) were also queried. The third section concerned analgesic medication usage (never used, $<1,1,2, \geq 3$ days/week), sleep status (excellent, good, fair, poor) and family history of headache.

ICHD-II [8] was applied to divide the patients into subgroups. Primary headache included migraine, TTH, cluster headache and other primary headaches [including primary stabbing headache, cough headache, hypnic headache, new daily persistent headache (NDPH) and others]. Secondary headache was diagnosed if the patient's headache could be attributed to viral infection, cranial neuralgias, cranial or cervical vascular disorder, psychiatric disorder, sinusitis or alcohol ingestion. If the headache could not be accurately categorized as either primary or secondary, it was classified as headache not otherwise specified (headache NOS).

Patients who reported a headache frequency of at least 15 days/month over a period lasting more than 3 months were classified as having chronic daily headache $(\mathrm{CDH})$. $\mathrm{CDH}$ included chronic migraine (CM), chronic tensiontype headache (CTTH), medication-overuse headache $(\mathrm{MOH})$, chronic cluster headache and NDPH. CM was defined by the following criteria revised by Olesen [9]: (1) headache (tension type and/or migraine) on $\geq 15$ days/ month for at least 3 months; (2) migraine that fulfilled ICDH-II and was experienced on $\geq 8$ days/month for at least 3 months; (3) no medication overuse.

For analysis, we defined the following two rules: (1) patients who fulfilled migraine diagnostic criteria, but also expressed other headache characteristics such as TTH, were categorized as having migraine; (2) probable migraine was also included with migraine. The study protocol was approved by the Ethical Committee at Chongqing Medical University and complied with the Declaration of Helsinki. All patients gave their informed consent for this study.

Statistical analysis of data was performed using the SPSS17.0 statistics package for PC. Demographic data were summarized using descriptive statistics. Quantitative data were presented as mean $\pm \mathrm{SD}$. The Student's $t$ test and the $\chi^{2}$ test were used for comparing quantitative and qualitative data, respectively. Separate variance estimation ( $t$ ' test) was used to detect possible differences in mean ages between primary headache and headache NOS, which had unequal variance. Mann-Whitney $U$ tests were performed for ordinal categorical variables to identify the clinical features differing between migraine and TTH. All 
Table 1 The gender and age distribution of headaches

* Age difference between migraine and TTH, $p<0.05$

\begin{tabular}{|c|c|c|c|c|}
\hline \multirow[t]{2}{*}{ Diagnosis } & \multirow[t]{2}{*}{ Age $($ mean \pm SD) } & \multicolumn{3}{|l|}{ Gender } \\
\hline & & Male N (\%) & Female $N(\%)$ & Both $N(\%)$ \\
\hline \multicolumn{5}{|l|}{ Primary headache } \\
\hline Migraine & $42.17 \pm 13.14^{*}$ & $78(19.5)$ & $323(80.5)$ & $401(23.8)$ \\
\hline TTH & $44.78 \pm 12.78$ & $132(33.1)$ & 267 (66.9) & $399(23.7)$ \\
\hline Cluster headache & $36.13 \pm 9.6$ & $7(87.5)$ & $1(12.5)$ & $8(0.5)$ \\
\hline Other headache & $43.91 \pm 15.48$ & $14(40.0)$ & $21(60.0)$ & $35(2.1)$ \\
\hline Secondary headache & $51.39 \pm 15.15$ & $146(32.2)$ & $308(67.8)$ & $454(27.0)$ \\
\hline Headache NOS & $46.02 \pm 15.45$ & $143(37.0)$ & $243(63.0)$ & $386(22.9)$ \\
\hline Total & $46.17 \pm 14.62$ & $520(30.9)$ & $1,163(69.1)$ & $1,683(100)$ \\
\hline
\end{tabular}

calculated $p$ values were two tailed and statistical significance was defined as a $p$ value of $<0.05$.

\section{Results}

Demographic characteristics of headache patients

Over the period of our study, 9,282 patients presented to the neurological outpatient department. Of these, 1,806 $(19.5 \%)$ reported headache as the primary reason for their visit. All these patients were interviewed; the questionnaire response rate was $93.19 \%(1,683 / 1,806)$. A total of 1,683 cases of headache patients were investigated; 640 (38\%) patients were seeking medical advice regarding their headache for the first time, $960(57.1 \%)$ patients had consulted for their headache previously and 83 (4.9\%) patients were unable to recall their headache consultation history. CT scan or MRI imaging to detect or exclude brain disorders was performed in $99(10.3 \%)$ of the 960 patients who had attended previously.

The age and gender distributions of the different headaches types are shown in Table 1. Primary headache patients accounted for $50.1 \%$ of all headache patients in our study, and migraine and TTH accounted for $23.8 \%$ $(401 / 1,683)$ and $23.7 \%(399 / 1,683)$ of the total, respectively. Secondary headache $(27 \%)$ was the next most frequently diagnosed headache type, and headache NOS accounted for $22.9 \%$.

Female patients $(69.1 \%, 1,163 / 1,683)$ predominated across all headache patients; there was no age difference between genders (males $45.95 \pm 15.69$ years, females $46.26 \pm 14.12$ years). Primary headache patients were generally younger than secondary headache and headache NOS patients $(43.42 \pm 13.11$ vs. $51.39 \pm 15.15$ years, $p<0.001 ; 43.42 \pm 13.11$ vs. $46.02 \pm 15.45$ years, $p=$ 0.004 by $t^{\prime}$ test). There was a clear pattern in the age distribution, with the number of primary headaches peaking in the age range 35-44 years. By contrast, the highest numbers of

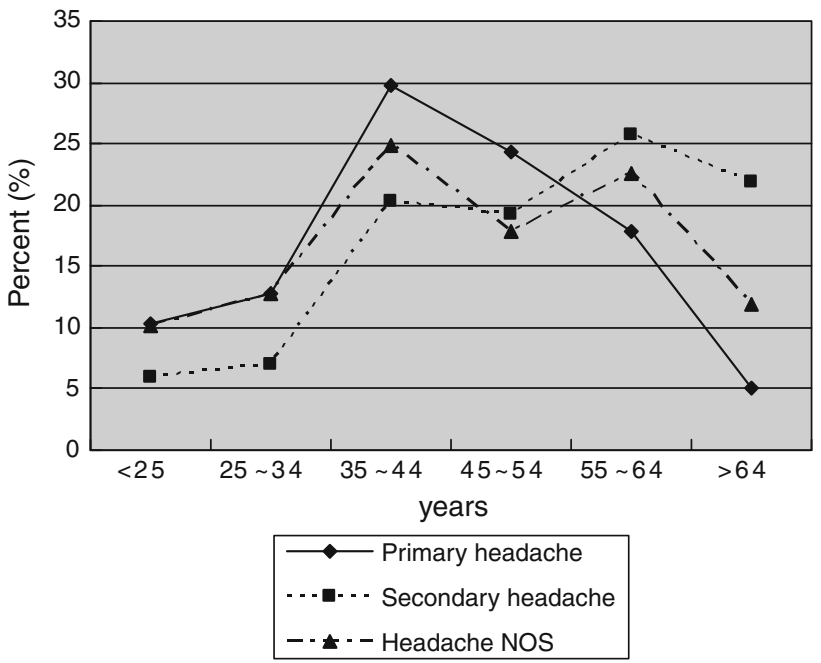

Fig. 1 Age distribution of headache patients

patients attending for secondary headache were in the age range 55-64 years (Fig. 1).

Clinical features of migraine and TTH

Of the patients of migraine, 215 (53.6\%) had migraine without aura; $163(40.7 \%)$ of the patients with probable migraine $23(5.7 \%)$ had migraine with aura and $58(14.5 \%)$ patients had migraine coexisting with TTH.

Of the study group, 68.6\% (267/389) of migraine patients and $64.9 \%(253 / 390)$ TTH patients had previously consulted for headaches $(p>0.05) ; 2.6 \% \quad(21 / 800)$ migraine and TTH patients were unable to recall their headache consultation history. Only $1.4 \%$ (11/800) had used preventive drugs, and this was flunarizine in all cases; none had used triptans for acute pain relief.

Although both migraine and TTH patients were predominantly in the age range of 35-44 years (Fig. 2), migraine patients were somewhat younger than TTH patients $\quad(42.17 \pm 13.14$ vs. $44.78 \pm 12.78$ years, $p<$ 0.05). Compared to TTH, there was a significantly higher 


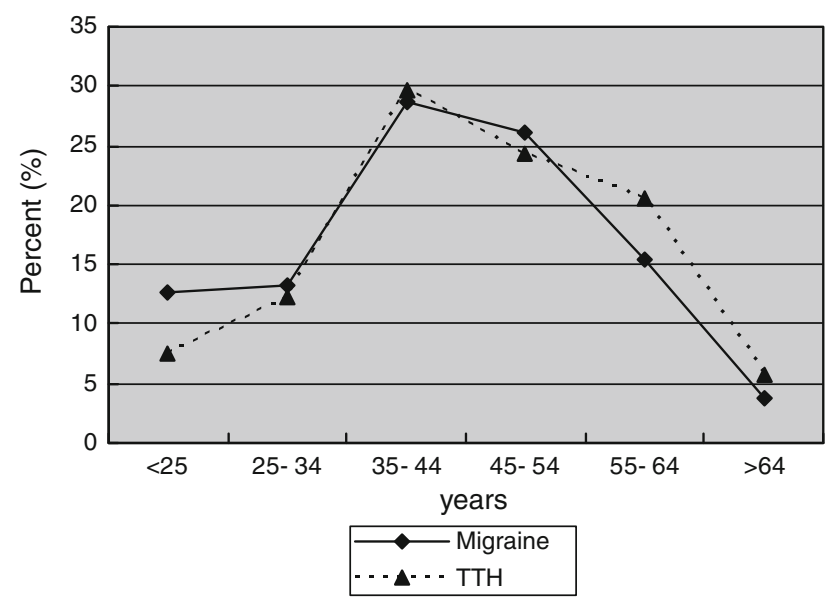

Fig. 2 Age distribution of migraine and TTH

proportion of females among the migraine patients $(80.5$ vs. $66.5 \%, p<0.001)$.

Table 2 presents the different clinical features of migraine and TTH headaches. Compared to TTH patients, migraine patients reported a longer duration of headache history, were more likely to use analgesic medication and experienced headache attacks of greater intensity. Further analysis showed that more migraine patients than TTH patients had a headache history of $\geq 6$ years (50.6 vs. $35.3 \%, p<0.001$ ), whereas the proportion of patients with a headache frequency $\geq 15$ days/month was higher in TTH patients than in the migraine group (54 vs. $35 \%, p<0.001$ ).
Chronic daily headache

Of the primary headache patients, $22.8 \%$ (192/843) were given an additional diagnosis of $\mathrm{CDH}$ by a headache specialist. As shown in Table 3, CTTH $(64.1 \%, 123 / 192)$ was the most common $\mathrm{CDH}$, followed by $\mathrm{MOH}(23.4 \%$, 45/192). Females also outnumbered males among the CDH patients (70.3 vs. $29.7 \%, p<0.001$ ), a gender ratio similar to that seen with episodic headaches. The mean age of $\mathrm{CDH}$ was higher than patients with episodic headache $(46.75 \pm 12.27$ vs. $42.45 \pm 13.26$ years, $p<0.001)$. The mean age of NDPH patients was the youngest, while MOH patients had a higher mean age than either NDPHs or CTTHs (Table 3).

Of the MOH patients, $20 \%(9 / 45)$ had primary headache of migraine, $75.6 \%(34 / 45) \mathrm{TTH}$, and 4.4\% (2/45) NDPH. With regard to the acute treatment drug types, $91.1 \%$ of $\mathrm{MOH}$ patients used combination analgesic medications, $8.9 \%$ used non-steroid anti-inflammatory drugs (NSAIDs) and none used triptans or ergot.

\section{Discussion}

In our survey, $19.5 \%$ patients gave headache as their primary reason for visiting the outpatient department. This is similar to the proportion of headache patients, 12.7 and $15.5 \%$, respectively, among patients to EDs in Italy [10] and Greece [3]. Because the health-care system in China
Table 2 Different clinical features between migraine and TTH

* Mann-Whitney $U$ test

\begin{tabular}{|c|c|c|c|c|c|}
\hline & Migraine $(N)$ & $\%$ & TTH $(N)$ & $\%$ & $p$ values \\
\hline \multicolumn{6}{|c|}{ Duration of headache history (years) } \\
\hline$<1$ & 55 & 13.7 & 70 & 17.5 & \multirow[t]{4}{*}{$<0.001 *$} \\
\hline $1-3$ & 79 & 19.7 & 126 & 31.6 & \\
\hline $4-6$ & 64 & 16.0 & 62 & 15.5 & \\
\hline$>6$ & 203 & 50.6 & 141 & 35.3 & \\
\hline Intensity & $6.29 \pm 1.34$ & & $4.22 \pm 1.52$ & & $<0.001$ \\
\hline \multicolumn{6}{|c|}{ Frequency (days/month) } \\
\hline$\leq 1$ & 100 & 26.10 & 50 & 13.00 & \multirow[t]{4}{*}{$<0.001 *$} \\
\hline $2-4$ & 63 & 16.40 & 49 & 12.70 & \\
\hline $5-14$ & 86 & 22.50 & 78 & 20.30 & \\
\hline$\geq 15$ & 134 & 35.00 & 208 & 54.00 & \\
\hline \multicolumn{6}{|c|}{ Analgesic medication uses (days/week) } \\
\hline No use & 173 & 43.1 & 234 & 58.6 & \multirow[t]{5}{*}{$<0.001 *$} \\
\hline$<1$ & 131 & 32.7 & 81 & 20.3 & \\
\hline 1 & 37 & 9.2 & 21 & 5.3 & \\
\hline 2 & 13 & 3.2 & 10 & 2.5 & \\
\hline$\geq 3$ & 47 & 11.7 & 53 & 13.3 & \\
\hline \multicolumn{6}{|c|}{ Family history } \\
\hline No & 235 & 59.3 & 295 & 75.4 & \multirow[t]{2}{*}{$<0.001$} \\
\hline Yes & 161 & 40.7 & 96 & 24.6 & \\
\hline
\end{tabular}


Table 3 Age and gender distribution of chronic daily headache (CDH)

\begin{tabular}{lccrc}
\hline CDH & Total $N(\%)$ & Male $N(\%)$ & Female $N(\%)$ & Age (mean \pm SD) \\
\hline CM & $9(4.7)$ & $3(33.3)$ & $6(66.5)$ & $48.89 \pm 7.72$ \\
CTTH & $123(64.1)$ & $35(28.5)$ & $88(71.5)$ & $46.38 \pm 12.19$ \\
MOH & $45(23.4)$ & $12(26.7)$ & $33(73.3)$ & $51.00 \pm 10.66^{*}$ \\
NDPH & $15(7.8)$ & $7(46.7)$ & $8(57.3)$ & $35.73 \pm 13.23^{* *}$ \\
Total & $192(100)$ & $57(29.7)$ & $135(70.3)$ & $46.75 \pm 12.27$ \\
\hline
\end{tabular}

* MOH patients were older than CTTHs and NDPHs $(p<0.05$, ANVOA analysis)

** NDPH patients were younger than other CDHs $(p<0.01$, ANVOA analysis $)$

Table 4 Headache diagnoses from different health-care departments (some data from Dermitzakis [3])

\begin{tabular}{lllllll}
\hline Diagnosis & $\begin{array}{l}\text { ED, Greek, } \\
2010(\%)[3]\end{array}$ & $\begin{array}{l}\text { ED, USA, } \\
\text { 2005 (\%) [12] }]\end{array}$ & $\begin{array}{l}\text { ED, Italy, } \\
\text { 2004 (\%) [10] }\end{array}$ & $\begin{array}{l}\text { Headache clinic, } \\
\text { USA 2011 (\%) [13] }\end{array}$ & $\begin{array}{l}\text { General medicine, } \\
\text { Japan 2010 }(\%)[11]\end{array}$ & $\begin{array}{l}\text { Neurology outpatient, } \\
\text { China 2010 }(\%)\end{array}$ \\
\hline Primary headache & 50.1 & 64 & 24.3 & 73.4 & 39.9 & 50.1 \\
Migraine & 15.4 & 38.7 & 16.7 & 51.4 & 30.8 & 23.8 \\
TTH & 33.6 & 7.1 & & 16 & 39.9 & 23.7 \\
Secondary headache & 22.1 & 25 & 41.3 & 9.4 & 42 & 27 \\
Headache NOS & 27.7 & 20 & 34.4 & 3.4 & 17.9 & 22.9 \\
\hline
\end{tabular}

permits patients to visit a medical center without referral from basic medical units, all patients were self-referred. Our outpatient study is therefore comparable to studies of patients attending EDs. However, a study in Japan which has a similar health-care system reported that only $8.9 \%$ of neurological outpatients gave headache as their primary reason for attending [11]. Our result showed that headache should be considered as one of the most important clinical issues for neurological outpatient physicians in china.

The percentage of secondary headache $(27 \%)$ in this study may be considered high. However, results of some other reports are similar to ours. Studies on ED patients found that $22.1 \%$ [3], 25\% [12] and $41.3 \%$ [10] of patients were attending for secondary headache. An outpatient headache clinic [13] and a general practice [11] reported 9.4 and $42 \%$, respectively (Table 4 ). We surmise that both the outpatient physician in our study and ED physicians are trained to recognize headache secondary to acute neurological disease first, whereas the diagnosis and subclassification of primary headache are primarily performed by headache clinics. It can often be difficult to distinguish between primary and secondary headaches, particularly in cases of acute headache and in patients aged more than 50 years. One study has suggested that headache patients older than 50 years have a fourfold higher risk that their headache is secondary to another predisposing condition [14]. Our results confirm that secondary headaches are more common than primary headaches in older patients.

Headache NOS $(22.9 \%)$ was also commonly encountered in our study. It is possible that this condition is over- represented because our survey was unable to provide a sufficiently detailed medical history and, upon further intensive investigation, only a proportion of these patients would be classified as headache NOS.

For primary headaches, migraine $(23.8 \%)$ and TTH $(23.7 \%)$ were the two most common headache types. Most previous studies have indicated that migraine was the most common headache type $[4,5,13]$. In previous reports from Asia, migraineurs represented the largest proportion of patients consulting their neurologists with a headache complaint $(50.9-85.8 \%)$ [5, 15]. It is possible that migraine is preferentially diagnosed by specialist of headache centers. However, an ED study in Greece [3] reported that TTH was the most frequent diagnosis for primary headache. Bendtsen [16] considered TTH to be the most common but also the most neglected type of headache. Our findings argue that TTH and migraine should be given equal attention in outpatient departments and EDs.

With regard to the age distribution of migraine and TTH, the present study showed that primary headache was most commonly encountered in patients aged 35-44 years. This contrasts with an epidemiological study of migraine in Taiwan [17] where the highest prevalence of primary headache was in the age range 30-34 years; in another study the majority of TTH patients were aged 30-39 years [1]. Overall, however, these findings demonstrate that migraine and TTH patients seeking medical attention for headache tend to be older than the average age of community patients.

For all types of headache, with the exception of cluster headaches, females were over-represented. For migraine, 
the female:male ratio was $4: 1$, a value consistent with reports that $79 \%$ of patients visiting an outpatient healthcare clinic [18] and $84 \%$ of patients attending a headache clinic [4] were female. For TTH patients in our study the female: male ratio was $2: 1$, whereas the general population data showed the ratio was 5:4 [19]. Overall, our data suggest that women are only marginally over-represented among TTH patients, whereas there was a significant gender bias in favor of females in patients with migraine.

\section{Different clinical features of migraine and TTH}

Although TTH and migraine can exhibit many similar features [20], respond effectively to similar medications and could possibly share similar genetic predisposing factors, there were clear differences between the TTH and migraine patients. Previous population-based studies reported that the average headache frequency was $<1$ day/ month in both migraine [17] and TTH [21]. However, in the present study, the majority of both migraine and TTH patients had headaches in excess of 1 day/month. Furthermore, the proportion of $\geq 15 \mathrm{day} /$ month headache in TTH patients was higher than in migraineurs. We also find that migraine patients attending the outpatient department have a longer duration of headache history than TTH patients, and also have both increased use of analgesic medications and a family history of headache. Our results support the contention from a general population study [22] that migraine and TTH are distinct entities, and that migraine is an all-or-none phenomenon triggered with an individually variable threshold, whereas the severity of TTH increases according to headache frequency. Our results suggest that headache intensity and duration of headache history are the most relevant factors for migraine patients, whereas headache frequency is the most important index of severity in TTH patients.

\section{Chronic daily headache}

Although $35 \%$ of migraine patients and $54 \%$ of TTH patients reported a headache frequency of $\geq 15$ days/ month, only $22.8 \%(192 / 1,683)$ of participants were defined as having $\mathrm{CDH}$. This reflects our criteria for $\mathrm{CDH}$ that require the patient not only to have a headache frequency of $\geq 15$ days/month, but also that the condition must have persisted for at least 3 months. CTTH was the most common condition, followed by MOH. Only $4.7 \%$ were defined to have CM. Similar clinical results were reported in Pakistan [15], while a study from India [23] found that $\mathrm{CM}$ was more common than CTTH. Using revised criteria for $\mathrm{CM}$, the Danish headache center diagnosed only $3 \%$ of patients with CM [24], arguing that the revised criteria were significantly more restrictive. $\mathrm{MOH}$ was common in patients with chronic headache, and they were generally older than non-MOH patients. The most common type of medication overuse was associated with the use of combination analgesic medications, which are easy to acquire and have a high risk of overuse, as previously reported in a study on Chinese people from Taiwan [25].

Limitations

The present study has several methodological limitations. First, because this is a hospital-based survey, the data cannot be used to estimate the prevalence of primary headaches in the general population. Second, the restrictive criteria for $\mathrm{CDH}$ employed in this study could underestimate the prevalence of this condition, most particularly of chronic migraine. Third, we did not evaluate the impact of the patient's condition on their work, household and social activities, and such factors should be taken into consideration when evaluating the wider impact of headache. Fourth, we compared only a limited number of clinical features in migraine and TTH; further studies will be required to differentiate between migraine and TTH and the specific factors that lead the patients to seek medical attention.

Acknowledgments The authors are grateful for the support by the Natural Science Foundation of China (Project No.30970988) and Chongqing Education Association (Project No.KJ100319).

\section{Conflict of interest None.}

Open Access This article is distributed under the terms of the Creative Commons Attribution License which permits any use, distribution and reproduction in any medium, provided the original author(s) and source are credited.

\section{References}

1. Stovner L, Hagen K, Jensen R, Katsarava Z, Lipton R, Scher A, Steiner T, Zwart JA (2007) The global burden of headache: a documentation of headache prevalence and disability worldwide. Cephalalgia 27(3):193-210. doi:10.1111/j.1468-2982.2007. 01288.x

2. Patterson VH, Esmonde TF (1993) Comparison of the handling of neurological outpatient referrals by general physicians and a neurologist. J Neurol Neurosurg Psychiatry 56(7):830

3. Dermitzakis EV, Georgiadis G, Rudolf J, Nikiforidou D, Kyriakidis P, Gravas I, Bouziani C, Tsiptsios I (2010) Headache patients in the emergency department of a Greek tertiary care hospital. J Headache Pain 11(2):123-128. doi:10.1007/s10194009-0178-3

4. Gesztelyi G, Bereczki D (2004) Primary headaches in an outpatient neurology headache clinic in East Hungary. Eur $\mathbf{J}$ Neurol 11(6):389-395. doi:10.1111/j.1468-1331.2004.00800.x

5. Wang SJ, Chung CS, Chankrachang S, Ravishankar K, Merican JS, Salazar G, Siow C, Cheung RT, Phanthumchinda K, Sakai F (2008) Migraine disability awareness campaign in Asia: migraine assessment for prophylaxis. Headache 48(9):1356-1365 
6. Lavados PM, Tenhamm E (2001) Consulting behaviour in migraine and tension-type headache sufferers: a population survey in Santiago, Chile. Cephalalgia 21(7):733-737. cha217 [pii]

7. Rasmussen BK, Jensen R, Olesen J (1992) Impact of headache on sickness absence and utilisation of medical services: a Danish population study. J Epidemiol Community Health 46(4):443-446

8. The International Classification of Headache Disorders: 2nd edition (2004). Cephalalgia 24 Suppl 1:9-160

9. Olesen J, Bousser MG, Diener HC, Dodick D, First M, Goadsby PJ, Gobel H, Lainez MJ, Lance JW, Lipton RB, Nappi G, Sakai F, Schoenen J, Silberstein SD, Steiner TJ (2006) New appendix criteria open for a broader concept of chronic migraine. Cephalalgia 26(6):742-746. doi:10.1111/j.1468-2982.2006.01172.x

10. Relja G, Granato A, Capozzoli F, Maggiore C, Catalan M, Pizzolato G, Zalukar W, Livia V, Gregorutti S, Zorzon M (2005) Nontraumatic headache in the Emergency Department: a survey in the province of Trieste. J Headache Pain 6(4):298-300. doi: 10.1007/s10194-005-0213-y

11. Okumura T, Tanno S, Ohhira M, Nozu T (2010) Characteristics in patients with headache in an outpatient clinic in Japan. Asia Pac Fam Med 9(1):10. doi:10.1186/1447-056X-9-10

12. Friedman BW, Hochberg ML, Esses D, Grosberg B, Corbo J, Toosi B, Meyer RH, Bijur PE, Lipton RB, Gallagher EJ (2007) Applying the International Classification of Headache Disorders to the emergency department: an assessment of reproducibility and the frequency with which a unique diagnosis can be assigned to every acute headache presentation. Ann Emerg Med 49(4): 409-419, 419 e401-409. doi:10.1016/j.annemergmed.2006.11. 004

13. Goldstein JN, Camargo CA Jr, Pelletier AJ, Edlow JA (2006) Headache in United States emergency departments: demographics, work-up and frequency of pathological diagnoses. Cephalalgia 26(6):684-690. doi:10.1111/j.1468-2982.2006.01093.x

14. Guerrero AL, Rojo E, Herrero S, Neri MJ, Bautista L, Penas ML, Cortijo E, Mulero P, Fernandez R (2011) Characteristics of the first 1,000 headaches in an outpatient headache clinic registry. Headache 51(2):226-231. doi:10.1111/j.1526-4610.2010.01828.x
15. Murtaza M, Kisat M, Daniel H, Sonawalla AB (2009) Classification and clinical features of headache disorders in Pakistan: a retrospective review of clinical data. PLoS One 4(6):e5827. doi: 10.1371/journal.pone.0005827

16. Bendtsen L, Jensen R (2006) Tension-type headache: the most common, but also the most neglected, headache disorder. Curr Opin Neurol 19(3):305-309. doi:10.1097/01.wco.0000227043. 00824.a9

17. Wang SJ, Fuh JL, Young YH, Lu SR, Shia BC (2000) Prevalence of migraine in Taipei, Taiwan: a population-based survey. Cephalalgia 20(6):566-572

18. Gibbs TS, Fleischer AB Jr, Feldman SR, Sam MC, O’Donovan CA (2003) Health care utilization in patients with migraine: demographics and patterns of care in the ambulatory setting. Headache 43(4):330-335. hed03067 [pii]

19. Jensen R, Stovner LJ (2008) Epidemiology and comorbidity of headache. Lancet Neurol 7(4):354-361. doi:10.1016/S1474-4422 (08)70062-0

20. Vargas BB (2008) Tension-type headache and migraine: two points on a continuum? Curr Pain Headache Rep 12(6):433-436

21. Lyngberg AC, Rasmussen BK, Jorgensen T, Jensen R (2005) Has the prevalence of migraine and tension-type headache changed over a 12-year period? A Danish population survey. Eur J Epidemiol 20(3):243-249

22. Rasmussen BK, Jensen R, Schroll M, Olesen J (1992) Interrelations between migraine and tension-type headache in the general population. Arch Neurol 49(9):914-918

23. Chakravarty A (2003) Chronic daily headaches: clinical profile in Indian patients. Cephalalgia 23(5):348-353

24. Zeeberg P, Olesen J, Jensen R (2009) Medication overuse headache and chronic migraine in a specialized headache centre: field-testing proposed new appendix criteria. Cephalalgia 29(2):214-220. doi:10.1111/j.1468-2982.2008.01710.x

25. Lu SR, Fuh JL, Chen WT, Juang KD, Wang SJ (2001) Chronic daily headache in Taipei, Taiwan: prevalence, follow-up and outcome predictors. Cephalalgia 21(10):980-986 